\title{
LES COLIBACILLES ET LES BACTÉRIOPHAGES DU LAIT DE CONSOMMATION EN NATURE A VARSOVIE (1)
}

\author{
par \\ IRÈNE LIPSKA \\ Institut Municipal d'Hygiène à Varsovie
}

Etant donné la grandé facilité d'avoir beaucoup d'échantillons de lait de marché apportés par des contrôleurs municipaux à l'Institut, pour l'analyse bactériologique (nombre de bactéries et titre de colibacilles), j'ai abordé l'étude de l'influence de la température atmosphérique et de la nourriture des vaches sur les espèces des colibacilles et sur les races des bactériophages actifs vis-à-vis du groupe coli-paratyphique du lait. J'ai choisi pour cette étude la fin de l'hiver et le commencement du printemps (le mois de mars et les premiers jours d'avril) et la fin de l'été et le commencement de l'automne (le mois d'août et les premiers jours de septembre). La première période fut caractérisée, en 1935, par la température minima de $-9^{\circ} \mathrm{C}$. et par $+3^{\circ} \mathrm{C}$. au maximum ; en cette saison les vaches sont mal nourries et ont peu de litière. A la seconde période, les conditions furent opposées : la température moyenne, aux jours choisis pour l'analyse, dépassait $25^{\circ} \mathrm{C}$, atteignant ainsi la température optima pour le développement des.microorganismes du lait, les vaches se trouvaient aux pâturages et les conditions de la production du lait étaient plus hygiéniques. Dix souches de colibacilles (colonies les plus typiques sur la gélose d'Endo) ont été isolées de dix échantillons du lait d'hiver et, dix fois, j'ai préparé des filtrats du bouillon ensemencé, avec tous les laits contrôlés ce jour-là, de manière que, pour $100 \mathrm{~cm}^{3}$ de bouillon, on ait ajouté $10 \mathrm{~cm}^{3}$ de lait. De même on a opéré dix fois en été; il faut souligner que les colibacilles etles filtrats pour bactériophages provenaient de différents échantillons du lait qui n'étaient pas analysés le même jour. On peut supposer que les colibacilles et les bactériophages isolés du lait de cette façon peuvent être considérés comme des représentants assez communs pour la saison. Le tableau I donne l'aperçu des propriétés biochimiques de dix souches de colibacilles d'hiver et de dix d'été. Dans le groupe d'hiver, le pouvoir fermentatif des souches est plus prononcé, on y trouve deux souches de Bact. neapolitanum, deux souches fermentant les einq substances hydrocarbonées, sauf le raffinose, deux souches de Bact. coli commune et deux souches attaquant les quatre substances (une ne touche pas la sorbite ni la

(1) Un rósumś de ce móm jire fut prósenté au Iİe Congrès international de microbiologie a Londres, 1936 . 
TABLEAU I.

\begin{tabular}{|c|c|c|c|c|c|c|c|c|c|c|c|c|c|c|}
\hline $\begin{array}{c}\text { Colibacilles } \\
\text { du lait }\end{array}$ & 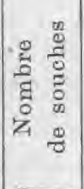 & 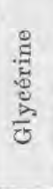 & $\begin{array}{l}\frac{5}{2} \\
\text { है } \\
\text { की }\end{array}$ & $\frac{\stackrel{0}{0}}{\frac{3}{3}}$ & 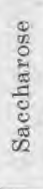 & 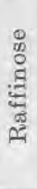 & 胥 & 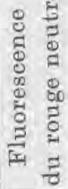 & 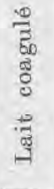 & $\begin{array}{l}\text { D2 } \\
\ddot{H}\end{array}$ & $\frac{\vec{\rho}}{\tilde{E}}$ & 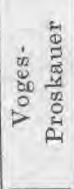 & 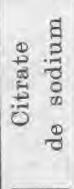 & 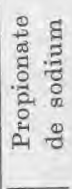 \\
\hline D'hiver .. & 10 & 10 & 9 & 7 & 6 & 4 & 7 & 9 & 10 & 8 & 4 & 3 & 8 & 3 \\
\hline D'étée. & 10 & 10 & 7 & 1 & 4 & 4 & 9 & 7 & 10 & 6 & 3 & 1 & 8 & 2 \\
\hline
\end{tabular}

dulcite et l'autre la dulcite et la salicine) ; une souche n'attaque que les trois premières substances et la dernière est le Bact. acidi lactici. Dans le groupe d'été, il y a deux souches de Bact. lactis aerogenes, une souche de Bact. coli commune, deux souches fermentant les quatre substances, sauf la sorbite et la dulcite, les trois souches n'attaquant que les trois premières substances et les deux dernières ne fermentant que les deux substances hydrocarbonées : une souche est le Bact. acidi lactici et l'autre attaque la glycérine et la salicine. De l'examen du tableau I ressort la faible fermentation de la dulcite dans le groupe d'été (une souche l'attaque) et le fait que la réaction de Voges-Proskauer est une fois positive. Il faut souligner que, parmi les dix souches d'hiver, il n'y en a que deux typiques (Bact. neapolitanum et Bact. coli commune) ; parmi les dix souches d'été il n'y a qu'une seule typique, c'est Bact. coli commune; toutes les autres présentent une discordance entre les quatre propriétés biochimiques essentielles : production d'indol et fermentation du propionate de sodium (caractères des colibacilles d'origine fécale), réaction de Voges-Proskauer positive et fermentation du citrate de sodium (caractères des colibacilles d'autre provenance). Ces résultats ressemblent à ceux obtenus avec les colibacilles isolés des déjections de nourrissons, il y a surtout une analogie entre les colibacilles des nourrissons malades (dyspepsie) et ceux des laits d'été ; dans les deux cas, la même faible fermentation de la dulcite et la réaction de Voges-Proskauer négative. Il faut aussi souligner la non fermentation de la sorbite ehez les trois souches de colibacilles d'été, la sorbite est d'habitude facilement attaquée par des colibacilles. J'ai déjà attiré l'attention sur le fait de la grande variabilité des souches de colibacilles du lait [2] ; ces souches d'origine fécale s'accoutument plus ou moins facilement à d'autres sources d'azote et de carbone, à la réaction souvent acide du milieu (surtout en été), à sa température plus basse et acquièrent avec le temps les caractères de Bact. lactis aerogenes. 
Passons maintenant aux résultats de l'étude des filtrats du lait faite sur les douze souches de colibacilles et sur les dix souches du groupe typhique et paratyphique, la vingt-troisième bactérie-test employée était Bact. dysenteriae Shiga. Les neuf premières souches de colibacilles provenaient des animaux marins invertébrés étudiés par moi en 1934 à Monaco [4] ; les trois dernières souches étaient isolées des déjections de l'homme sain $\left(n^{\circ s} 10\right)$ et de malades ( ${ }^{0 s} 11$ et 12). Quant aux bactéries du groupe typhique et paratyphique, ce sont les mêmes souches sensibles du laboratoire employées par moi dans mon étude sur les typhophages du lait [3]. Les tableaux II et III donnent le comportement des dix filtrats du lait d'hiver et des

TABLEAU II.

\begin{tabular}{|c|c|c|c|c|c|c|c|c|c|c|c|c|c|c|}
\hline \multirow[b]{2}{*}{$\begin{array}{l}\text { Filtrats du } \\
\text { lait d'hiver }\end{array}$} & \multicolumn{12}{|c|}{ Colibacilles } & \multirow{2}{*}{ 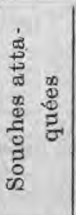 } & \multirow{2}{*}{ 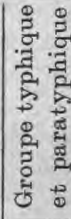 } \\
\hline & 1 & 2 & 3 & 4 & 5 & 6 & 7 & 8 & 9 & 10 & 11 & 12 & & \\
\hline \multirow{11}{*}{$\begin{array}{l}1 \\
2 \\
2\end{array} \ldots \ldots$} & & + & + & & + & - & + & + & & + & + & + & 8 & 8 \\
\hline & & + & & & & t & & & & + & + & + & 5 & 6 \\
\hline & & & I & & & & + & + & & + & + & & 4 & 5 \\
\hline & & & & & & + & + & + & + & + & & & 5 & 5 \\
\hline & & & + & & & & & & + & & & & 2 & 2 \\
\hline & & + & + & & & & & & + & & & & 3 & 3 \\
\hline & & & & + & & + & + & & & + & + & & 5 & 5 \\
\hline & & + & . & & & + & + & & + & + & & + & 6 & 5 \\
\hline & & + & + & & & & + & & & + & & + & 5 & 4 \\
\hline & & & + & & & & + & & & + & + & & 4 & 5 \\
\hline & & 5 & 5 & 1 & 1 & 4 & 7 & 3 & 4 & 8 & 5 & 4 & 47 & 48 \\
\hline
\end{tabular}

dix de ceux d'été vis-à-vis des vingt-trois bactéries-tests. On voit que, dans tous les filtrats, on peut déceler des bactériophages actifs si l'on emploie un nombre assez grand de bactéries sensibles et si l'on a soin de faire la double épreuve du bactériophage : l'émulsion des bactéries sur du bouillon dans les tubes placés à l'étuve à $32^{\circ} \mathrm{C}$. et l'étalement du liquide sur la gélose à $1 \%$ à $22^{\circ} \mathrm{C}$.; de cette manière, les résultats ne peuvent pas être faussés par la formation des cultures secondaires de bactéries résistantes et on peut reconnaitre la présence des bactériophages même peu actifs par la formation des plages sur la gélose inclinée. L'examen comparatif de ces deux groupes de filtrats montre que l'étendue de leur virulence varie 
TABLEAU III.

\begin{tabular}{|c|c|c|c|c|c|c|c|c|c|c|c|c|c|c|}
\hline \multirow{2}{*}{$\begin{array}{l}\text { Filtrats du } \\
\text { lait d'été }\end{array}$} & \multicolumn{12}{|c|}{ Colibacilles } & \multirow{2}{*}{ 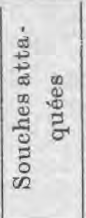 } & \multirow{2}{*}{ 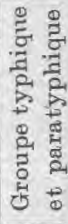 } \\
\hline & 1 & 2 & 3 & 4 & 5 & 6 & 7 & 8 & 9 & 10 & 11 & 12 & & \\
\hline $1 \ldots \ldots \ldots$ & & + & + & & + & & + & + & & + & & & 6 & 2 \\
\hline $2 \ldots \ldots \ldots$ & & + & 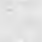 & & + & & & & & + & + & + & 5 & 4 \\
\hline $3 \ldots \ldots \ldots$ & & + & + & + & + & 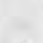 & + & & & 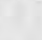 & + & & 6 & 6 \\
\hline $4 \ldots \ldots \ldots$ & & + & + & + & + & + & & + & + & & + & & 8 & 7 \\
\hline $5 \ldots \ldots \ldots$ & & + & & 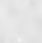 & & & + & & & + & & & 3 & $t$ \\
\hline $6 \ldots \ldots \ldots$ & & - & & + & 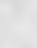 & 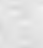 & & & & + & + & & 3 & 4 \\
\hline $7 \ldots \ldots \ldots$ & & & & . & & & & & & + & + & & 2 & \\
\hline $8 \ldots \ldots \ldots$ & + & & + & + & 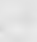 & & + & + & & + & + & + & 8 & 5 \\
\hline $9 \ldots \ldots \ldots$ & & + & + & + & 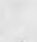 & & + & + & & + & + & & 7 & 6 \\
\hline $10 \ldots \ldots \ldots$ & & + & + & + & + & & + & & & & + & & 6 & 4 \\
\hline$x$ & 1 & 7 & 6 & 6 & 5 & 1 & 6 & 4 & 1 & 7 & 8 & 2 & 54 & 47 \\
\hline
\end{tabular}

depuis quatre souches attaquées jusqu'à seize ; de même, la force de la virulence diffère d'un filtrat à l'autre et d'une souche bactérienne à l'autre depuis un simple retard jusqu'à l'arrêt de croissance dans le cas du bouillon légèrement ensemencé ; depuis une dissolution à peine perceptible de l'émulsion jusqu'à une dissolution totale des bactéries ; depuis une nappe sur gélose avec une à dix plages jusqu'aux débris de culture ou des colonies isolées, parfois la gélose reste complètement stérile. Dans le groupe de filtrats du lait d'hiver la souche humaine $\mathrm{n}^{0} 10$ était la plus sensible (elle était huit fois attaquée) ; la souche animale $n^{0} 7$ était un peu moins sensible (7 fois attaquée). La sensibilité de souches de colibacilles vis-à-vis des filtrats du lait d'été était plus grande, elles étaient attaquées en somme cinquante-quatre fois contre quarante-sept fois du premier groupe. Il faut noter, que ce ne sont pas les mêmes souches qui se sont montrées les plus sensibles dans les deux groupes de filtrats du lait (voir les colibacilles $n^{0} 4$ et $n^{0} 9$ ). L'action des filtrats du lait s'exerce avec la même intensité sur les colibacilles et sur les bacilles typhiques et paratyphiques: l'étendue et la force de la virulence d'un filtrat sont respectivement grandes ou petites pour les souches de ces deux groupes de bacilles. Après avoir constaté la plus ou moins grande activité des filtrats du lait pour les colibacilles d'origine fécale animale et humaine, j'ai voulu étudier leur action sur les vingt eolibacilles du lait d'hiver et d'été. Cet examen fut fait après deux à 
huit mois de la conservation à la glacière des filtrats du lait et de la culture des colibacilles sur la gélose de la même durée. Les résultats de cette épreuve sont les suivants : les deux filtrats du lait d'hiver ( $\mathrm{n}^{\circ} 3$ et $\mathrm{n}^{0} 4$ ) se sont montrés faiblement actifs einq fois pour les colibacilles d'hiver et six fois pour ceux d'été ; les trois filtrats du lait d'été ( $\mathrm{n}^{0 \mathrm{~s}} 2,4$ et 9 ) ont attaqué dix fois les colibacilles du lait d'hiver avec une formation des plages huit fois et quatorze fois ceux du lait d'été, les plages de 1 à $2 \mathrm{~mm}$. furent observées cinq fois ; de nouveau les filtrats du lait d'été furent plus actifs que ceux du lait d'hiver. Il faut souligner, qu'une même souche des colibacilles du lait d'hiver fut attaquée par les trois filtrats du lait d'été et par les deux filtrats du lait d'hiver ; cette constatation prouve bien que la-sensiblité des souches d'une même espèce joue un rôle très important dans l'étude de la bactériophagie.

Pour étudier l'action des filtrats du lait sur les colibacilles pathogènes, $j$ 'ai isolé dix souches de l'urine infectée des malades atteints des affections rénales. J'ai effectué les expériences en utilisant deux filtrats du lait d'hiver et aussi deux d'été, en choisissant les plus actifs, et j'ai observé, avec un filtrat d'été, une dissolution presque complète d'une émulsion en bouillon d'une culture jeune sur gélose de deux souches de l'urine, avec formation des plages; un autre filtrat d'été a produit une seule fois un éclaircissement de l'émulsion. Les filtrats du lait d'hiver furent complètement inactifs pour ces colibacilles. Cette faible action des filtrats du lait sur les colibacilles pathogènes peut être expliquée par le fait encore fort discuté de leur spécifjcité, voir les mémoires de Teveli [5] et de Hassmann [1].

\section{CONCLUSIONS}

1. Les colibacilles du lait d'hiver ainsi que ceux du lait d'été sont caractérisés par leurs propriétés biochimiques intermédiaires entre les espèces Bact. coli commune et Bact. lactis aerogenes.

2. Les filtrats du lait d'été sont plus actifs vis-à-vis des colibacilles isolés du lait, des déjections animales et humaines et des urines des malades atteints d'affections rénales, que ceux du lait d'hiver.

3. On observe une analogie dans l'activité des filtrats du lait pour les colibacilles et pour les bacilles typhiques et paratyphiques.

4. La plus grande étendue de l'activité des filtrats du lait d'été embrassait seize souches sur les vingt-trois bacilles-tests.

5. Les plus souvent attaqués étaient les colibacilles des déjections animales et humaines, ceux du lait étaient plus résistants, et, les plus réfractaires étaient les colibacilles pathogènes, isolés de l'urine. 


\title{
BIBLIOGRAPHIE
}

[1] K. Hassmann. Centralbl. f. Bakt. Refer., 1935, 116, 278.

[2] I. Lipska. Le Lait, 1934, 14, 673.

[3] I. LIPSKa. $X^{\mathrm{e}}$ Congrès mondial de Laiterie, Rome, 1934. II ${ }^{\mathrm{e}}$ Section, p. 245 .

[4] 1. Lipska. Bulletin de l'Institut Océanographique de Monaco, 1935, n 675.

[5] Z. Teveli. Oentralbl. f. Bakt. Refer., 1932, 108, 43.

\section{LA PRODUCTION D'UN LAIT PROPRE ET SAIN A LA FERIME (1)}

\author{
par
}

\section{A. TAPERNOUX}

Professeur de Chimie à l'Ecole Nationale Vétérinaire de Lyon

\begin{abstract}
"Il y a un magnifique programme à remplir, car améliorer la qualité du lait et des produits qu'on en retire, c'est tout à la fois répondre aux indications d'une hygiène qui, à bon droit, se fait tous les jours plus exigeante, et c'est contribuer également au développement de la richesse publique."
\end{abstract}

Ch. Poвсher, Le Lait, 1921, nº 1 .

Monsieur le Président,

Mesdames, Messieurs,

Qu'il me soit permis tout d'abord de féliciter les Organisateurs de la Semaine du Lait de Grenoble, à l'occasion de la Foire, en faveur de la production, de la consommation du lait et de ses dérivés.

Problème économique, d'une grande importance pour l'agriculture nationale, et il s'est présenté naguère sous un aspect si angoissant que le Gouvernement et le Parlement sont intervenus par la loi du 2 juillet 1935 qui a cherché à assainir le marché du lait. Pour obtenir ce résultat, il faut que les producteurs soient bien convaincus qu'il n'y a qu'un moyen : produire un lait propre et sain.

Dès qu'on présente au public, en effet, du lait donnant une sécurité complète, la consommation augmente.

Je suis heureux de venir défendre ici un certain nombre d'idées qui me sont chères parce qu'elles m'ont été inculquées par mon Maître, le regretté Professeur Ch. PoRcher ; et c'est pourquoi j'adresse l'expression de ma reconnaissance à M. PIRAuD, président du Comité d'Organisation, à $\mathbf{M}$. Roy, directeur des Services agricoles du département de l'Isère, secrétaire général du Comité, au $\mathrm{D}^{\mathrm{r}} \mathrm{M}_{\mathrm{A}}$ -

(1) Conférence donnée le samedi 29 août 1936 à la Chambre de Commerce de Grenoble, sous les auspices du Comité d'Organisation de la Semaine du Lait. 